QUARTERLY OF APPLIED MATHEMATICS

VOLUME LXV, NUMBER 2

JUNE 2007, PAGES 375-383

S $0033-569 X(07) 01048-7$

Article electronically published on April 19, 2007

\title{
DERIVATION OF EIGENRELATIONS FOR THE STURM-LIOUVILLE BOUNDARY VALUE PROBLEMS WITH INTERIOR SINGULARITIES
}

BY

THOMAS M. ACHO

Department of Mathematics and Applied Mathematics, University of the Free State, P.O. Box 339

Bloemfontein 9300, Republic of South Africa

\begin{abstract}
Asymptotic solutions for the Sturm-Liouville boundary value problem with interior singularities were obtained using asymptotic forms of the Whittaker functions for higher order modes and the Titchmarsh-Weyl $m$-function for low order modes. However, these split interval techniques do not readily provide the eigenrelations for low order modes. For the first time, with minimal constraints, the eigenvalues for the SturmLiouville eigenproblem are obtained when the Titchmarsh-Weyl $m$-function technique is employed.
\end{abstract}

1. Introduction. Numerous studies have been carried out geared towards obtaining asymptotic solutions for the Sturm-Liouville (SL) eigenproblems which occur in quantum mechanics, duirnal ocean tides and planetary waves where a strong singularity other than the simple pole is much more evident. Methods of solutions have been developed in 4, [5], 11, [12, 13, 17, 18, and more recently in 11, 2], 3]. It has been shown that the SL boundary value problem with interior double pole and two turning points has complex eigenvalues. A physical implication of complex eigenvalues ([6], [7]) is that the models of planetary waves on a sphere propagate vertically, while their density weighted fields are simultaneously decaying with height. This self-adjoint SL eigenproblem may be given as

$$
\begin{gathered}
\frac{d^{2} U}{d \xi^{2}}+P(\xi) U=0, \quad I_{\xi}=[a, b] \\
U(a)=U(b)=0, \quad a<0<b
\end{gathered}
$$

Received June 13, 2006 and, in revised form, September 22, 2006.

2000 Mathematics Subject Classification. Primary 54B24, 34L20; Secondary 34E20, 33C15, 34E05.

Key words and phrases. Asymptotic approximation, interior singularities, turning points, Whittaker functions, Titchmarsh-Weyl $m$-function, direct sum method, double pole, split interval, eigenfunction solutions, eigenvalue relations, high order modes, low order modes.

E-mail address: achotm.blms@mail.uovs.ac.za 
where $P(\xi)$ has a double pole and two simple turning points as the only transition points. As indicated in [2], the standard SL theory permits singularities only at the end points of the interval $I_{\xi}$ for $P(\xi)$ real and continuous on $I_{\xi}$. In this case the eigenvalues are real and eigenfunctions exist even if there are poles at either $a$ or $b$. However when we consider the non-standard SL eigenproblems which have interior singularities then (1.1) with (1.2) has complex eigenvalues. Solutions for the SL boundary value problem (BVP) with an interior simple pole was presented by Boyd [5] and for interior double poles with turning points by Clemence and Acho [2, and using matched asymptotic technique by Acho [1]. However, computation of the eigenvalues of the SL BVP when the TitchmarshWeyl $m$-function theory (we refer the reader to whom notion of the Titchmarsh-Weyl $m$-function theory is unfamiliar to [10]) is used will be presented for the first time in this paper. For the sake of brevity, results already obtained in [1] and 2] will be cited, but details of the derivation will not be presented in this paper.

However, the necessary analysis for obtaining the solution of the SL eigenproblem as obtained in [2] is presented in section 2 with the necessary theorems (proofs in 2] are omitted here), and in section 3 we compute the eigenvalues.

\section{Solutions of the SL boundary value problem.}

2.1. Preliminaries. For a clear understanding of the representation of the asymptotic solutions we will re-write the SL eigenproblem (1.1) with (1.2) in the form

$$
\begin{gathered}
\frac{d^{2} U}{d \xi^{2}}+\frac{\gamma_{0}+\gamma_{1} \xi+\gamma_{2} \xi^{2}}{\xi^{2}} U=0, \quad I_{\xi}=[a, b], \\
U(a)=U(b)=0, \quad a<0<b,
\end{gathered}
$$

which has turning points

$$
\tau_{1,2}=\frac{-\gamma_{1} \pm \sqrt{\gamma_{1}^{2}-4 \gamma_{0} \gamma_{2}}}{2 \gamma_{2}}
$$

where $\gamma_{2}$ are eigenvalues.

We expect the eigenfunction solutions of our SL eigenproblem (2.1) with (2.2) to be Whittaker functions $M_{k, \mu}\left(2 i \gamma_{2}^{1 / 2} \xi\right)$ and $W_{k, \mu}\left(2 i \gamma_{2}^{1 / 2} \xi\right)$. The asymptotic expansions for $\left|2 i \gamma_{2}^{1 / 2} \xi\right|$ large and $\gamma_{2} \rightarrow \infty$ along the negative imaginary axis are given by

$$
\frac{\Gamma(1 / 2+\mu)}{\Gamma(1+2 \mu)} M_{k, \mu}\left(2 i \gamma_{2}^{1 / 2} \xi\right) \approx e^{\left[i \gamma_{2}^{1 / 2} \xi\right]}+e^{-i\left[\gamma_{2}^{1 / 2} \xi+\pi(\mu+1 / 2)\right]}
$$

and

$$
W_{k, \mu}\left(2 i \gamma_{2}^{1 / 2} \xi\right) \approx e^{-i \gamma_{2}^{1 / 2} \xi},
$$

where $M_{k, \mu}$ and $W_{k, \mu}$ are Whittaker functions and

$$
k=\frac{-i \gamma_{1}}{2 \gamma_{2}^{1 / 2}}, \quad \mu=\left(1 / 4-\gamma_{0}\right)^{1 / 2} .
$$

Thus from (2.3) and (2.4) we obtain the asymptotic solution (we refer the reader to whom the notion of asymptotics is unfamiliar to [8, 15 ) for higher order modes as

$$
w\left(\xi, \gamma_{2}\right)=\alpha_{h} \cos \left(\left|\gamma_{2}\right|^{1 / 2} \xi\right)+\beta_{h} \sin \left(\left|\gamma_{2}\right|^{1 / 2} \xi\right)
$$


where

$$
\begin{aligned}
\alpha_{h} & =\alpha_{0}\left\{1+e^{i \pi(\mu+1 / 2)}\right\}+\beta_{0}, \\
\beta_{h} & =i\left\{\alpha_{0}\left(1+e^{-i \pi(\mu+1 / 2)}\right\}-\beta_{0},\right.
\end{aligned}
$$

and $\alpha_{0}, \beta_{0}$ are complex constants.

For $\left|\gamma_{2}\right|$ small, an internal boundary layer in which available asymptotic series are inaccurate include the whole of $[a, b]$. This is a consequence of the presence of the double pole for $\left|\gamma_{2}\right|$ small, especially when the turning points are separated.

The method of finding asymptotic approximations to solutions (2.1) with (2.2) would be identical to that of the simple pole case if both signs of the real part of the turning points are the same. In this case, we have at most two of the zeros of the Whittaker functions from which to choose one to satisfy boundary condition at $b$, while for the interval $(a, 0)$ we invoke theorem 3 of [13. We shall however dwell on the case where sign of the real part of the turning points are different and separated, that is, when $|Q|<|T|$ and $|R|<|I|$, where

$$
\begin{aligned}
Q & =\operatorname{Im} \gamma_{2} \cdot \operatorname{Re}\left\{-\gamma_{1} \pm \sqrt{\gamma_{1}^{2}-4 \gamma_{0} \gamma_{2}}\right\} \\
T & =\operatorname{Re} \gamma_{2} \cdot \operatorname{Im}\left\{-\gamma_{1} \pm \sqrt{\gamma_{1}^{2}-4 \gamma_{0} \gamma_{2}}\right\} \\
R & =\boldsymbol{\operatorname { R e }} \gamma_{2} \cdot \operatorname{Re}\left\{-\gamma_{1} \pm \sqrt{\gamma_{1}^{2}-4 \gamma_{0} \gamma_{2}}\right\} \\
I & =\operatorname{Im} \gamma_{2} \cdot \operatorname{Im}\left\{-\gamma_{1} \pm \sqrt{\gamma_{1}^{2}-4 \gamma_{0} \gamma_{2}}\right\},
\end{aligned}
$$

where Im stands for Imaginary part, and Re for Real part.

Figures 1- 4 show the positions of the turning points.

The approach in [1] is to obtain an inner expansion as $\left|2 i \gamma_{2}^{1 / 2} \xi\right| \rightarrow \infty$ about the turning point $\tau_{1}$, whose real part is greater than zero. This would be matched to the outer asymptotic expansion for $\xi \rightarrow \tau_{1}$ along the anti-Stokes lines $A_{1}$ (if we appeal to Figure 2, say). The result from the matching would then be used to satisfy a boundary condition at $b$. For a boundary condition at $a$, we shall use the analytic continuation (a connection formula) for these functions about the double pole for $\xi=\xi e^{\pi i}$. Again, the result of the matching will then be used to satisfy a boundary condition at $a$.

The asymptotic series for $\left|\gamma_{2}\right|$ small (asymptotic series of the Whittaker functions for $|\gamma|$ small and $4 k=x$ or $4 k \neq x$ as given in [19] do not represent the turning point as in the simple pole case of [5]) are not valid around the turning points in the double pole case. This necessitated the use of WKB approximation as the outer expansion and the Airy approximation as the inner expansion about each turning point.

The WKB approximation for the general solutions to (2.1) are given by

$$
\begin{aligned}
& w_{1}=[\nu(\xi)]^{-1 / 4} \exp \left\{-i \int_{0}^{\xi}[\nu(s)]^{1 / 2} d s\right\}, \\
& w_{2}=[\nu(\xi)]^{-1 / 4} \exp \left\{i \int_{0}^{\xi}[\nu(s)]^{1 / 2} d s\right\}
\end{aligned}
$$


In Figs 1 - 4: Aj \& Aj' , j=1,2,3 ARE anti-Stokes lines ; $\mathrm{Sj} \& \mathrm{Sj}{ }^{\prime}, \mathrm{j}=1,2,3$ ARE STOKES LINES; AND $\bigcirc$ zEROS OF EIGENFUNCTIONS

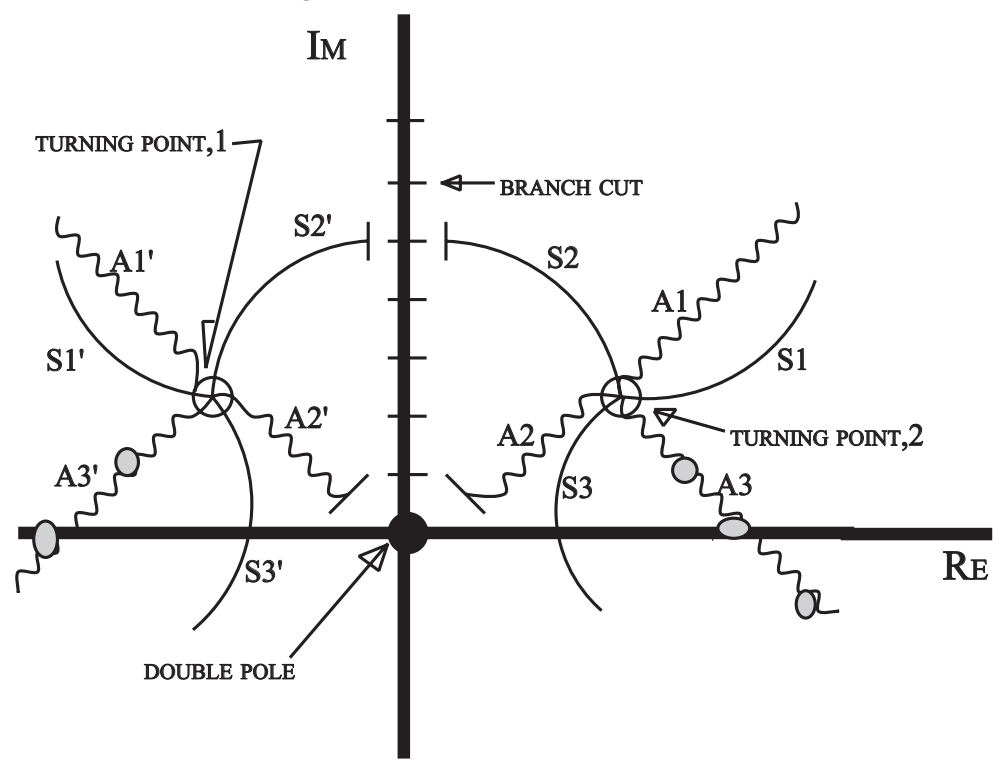

FIG 1: TURNING POINTS BOTH LIE IN THE UPPER HALF OF THE COMPLEX PLANE AND TWO OF THE ZEROS OF THE EIGENFUNCTIONS LIE ON THE REAL AXIS.

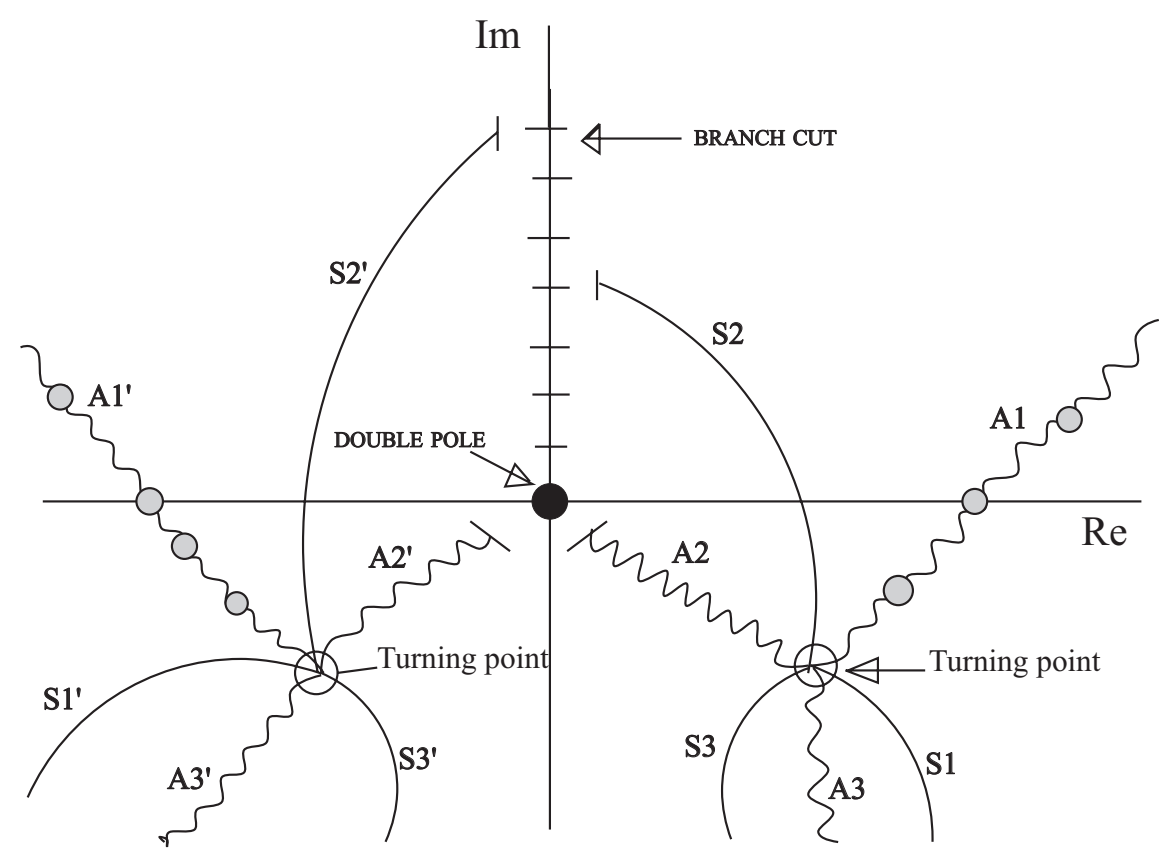

Fig 2: TURNING POINTS Both LIE IN THE LOWER HALF PLANE AND TWO OF THE ZEROS OF THE EIGENFUNCTIONS LIE ON THE REAL AXIS. 


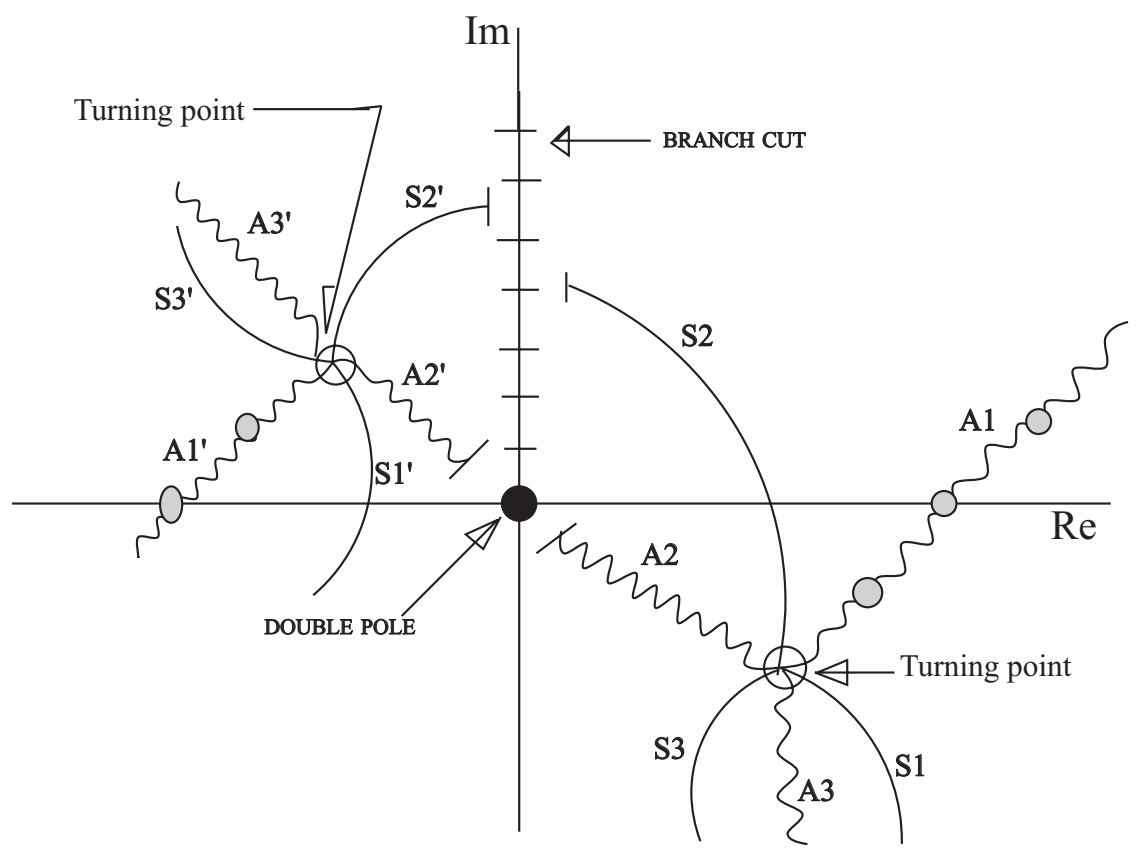

Fig 3: One of the turning points lies in the lower half of the complex plane and the other in the upper half plane.

where

$$
\nu(s)=\frac{\gamma_{0}+\gamma_{1} s+\gamma_{2} s^{2}}{s^{2}}
$$

The separated matching that follows in [1] would then yield the eigenrelation.

The solution and eigenrelations to the SL BVP for high order modes were obtained for the double pole case in [1] and [2] and for the simple pole case in [5].

2.2. Titchmarsh-Weyl m-function theory eigenfunction solution for low order modes. The relatively simpler split interval technique employed in [2] uses Titchmarsh-Weyl $m$-function theory [10, for which the boundary conditions are automatically satisfied by the solution obtained. Here the asymptotic analysis of the eigenfunctions (i.e., the Whittaker functions) is also as presented above. With preliminary WKB analysis the illustrative diagrams above show that the transition points lie in the $\xi$-complex plane with nonzero imaginary parts. This helps establish the convenient split intervals necessary for the Titchmarsh-Weyl $m$-function theory, the splits being appropriately located at the turning points, which lie on antistokes lines, 2. This is particularly important when we consider the fact that for some SL eigenproblems (notably those on planetaryscale stratospheric disturbances [7, [1], 18]), these transition points represent singular lines. 


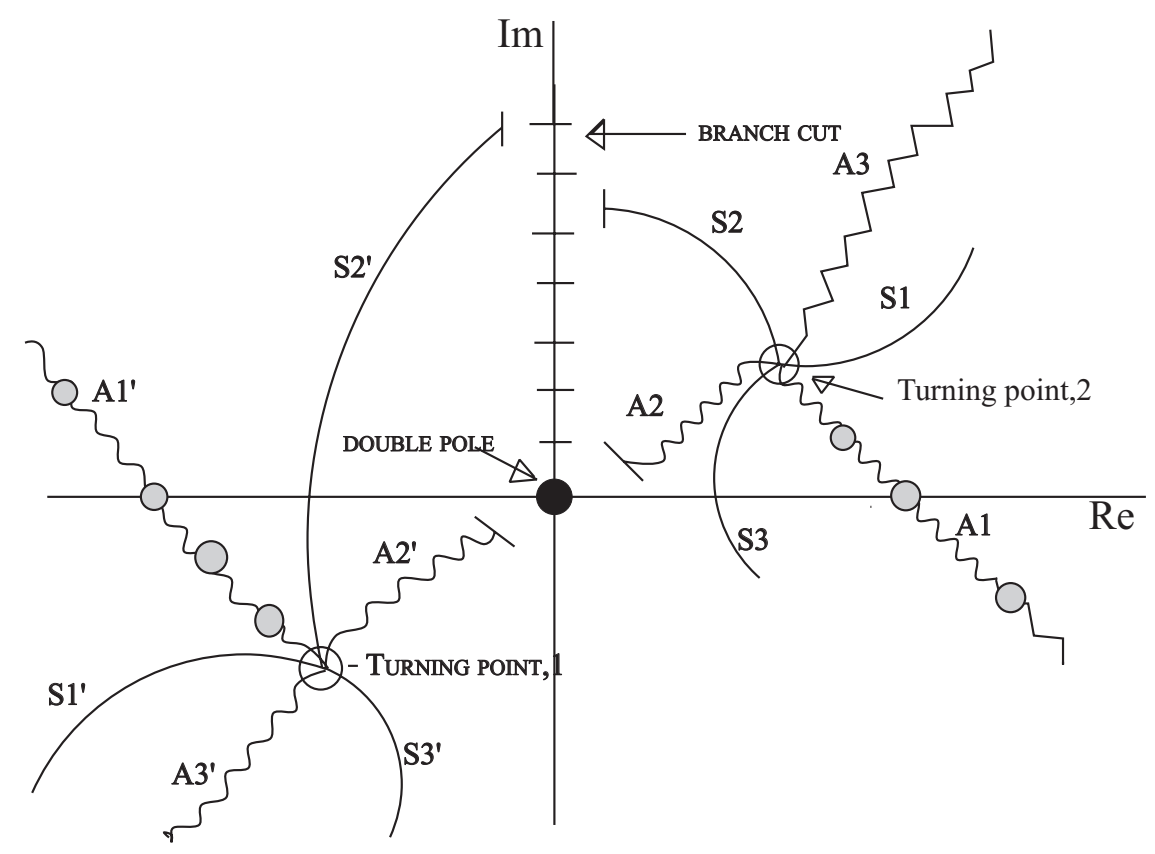

FIG 4: ONE OF THE TURNING POINTS LIES IN THE LOWER HALF OF THE COMPLEX PLANE AND THE OTHER IN THE UPPER HALF PLANE.

The solution to the SL BVP for low order modes using the Titchmarsh-Weyl $m$ function technique (as in 2]) is

$$
U\left(\xi, \gamma_{2}\right)=\theta\left(\xi, \gamma_{2}\right)+\left\{\begin{array}{l}
m^{+}\left(\gamma_{2}\right) \phi\left(\xi, \gamma_{2}\right) \in L^{2}\left(\left[\tau_{1}^{*}, b\right]\right), \\
m^{-}\left(\gamma_{2}\right) \phi\left(\xi, \gamma_{2}\right) \in L^{2}\left(\left(0, \tau_{1}\right)\right), \\
n^{+}\left(\gamma_{2}\right) \phi\left(\xi, \gamma_{2}\right) \in L^{2}\left(\left(\tau_{2}^{*}, 0\right)\right), \\
n^{-}\left(\gamma_{2}\right) \phi\left(\xi, \gamma_{2}\right) \in L^{2}\left(\left[a, \tau_{2}\right)\right),
\end{array}\right.
$$

where

$$
\begin{aligned}
& m^{+}\left(\gamma_{2}\right)=-\operatorname{Lim}_{\xi \rightarrow b} \frac{\theta\left(\xi, \gamma_{2}\right)}{\phi\left(\xi, \gamma_{2}\right)}, \\
& m^{-}\left(\gamma_{2}\right)=\underset{\xi \rightarrow 0^{+}}{-\operatorname{Lim}} \frac{\theta\left(\xi, \gamma_{2}\right)}{\phi\left(\xi, \gamma_{2}\right)}, \\
& n^{-}\left(\gamma_{2}\right)=\underset{\xi \rightarrow 0^{-}}{-\operatorname{Lim}} \frac{\theta\left(\xi, \gamma_{2}\right)}{\phi\left(\xi, \gamma_{2}\right)}, \\
& n^{+}\left(\gamma_{2}\right)=\underset{\xi \rightarrow a}{-\operatorname{Lim}} \frac{\theta\left(\xi, \gamma_{2}\right)}{\phi\left(\xi, \gamma_{2}\right)},
\end{aligned}
$$

and

$$
\begin{gathered}
\theta\left(\xi, \gamma_{2}\right)=\alpha_{0} M_{k, \mu}\left(2 i \gamma_{2}^{\frac{1}{2}} \xi\right)+\alpha_{1} M_{k,-\mu}\left(2 i \gamma_{2}^{\frac{1}{2}} \xi\right) \\
\phi\left(\xi, \gamma_{2}\right)=\beta_{0} W_{k, \mu}\left(2 i \gamma_{2}^{\frac{1}{2}} \xi\right)+\beta_{1} W_{-k, \mu}\left(-2 i \gamma_{2}^{\frac{1}{2}} \xi\right)
\end{gathered}
$$


where $M_{k, \mu}, M_{k,-\mu}, W_{k, \mu}$ and $W_{-k, \mu}$ are Whittaker functions, with $\alpha_{0}, \alpha_{1}, \beta_{0}, \beta_{1}$, as obtained in section 3 of [2]. The eigenrelation for the low order modes will be obtained in the section that follows.

3. Derivation of the eigenrelation for low order modes. As with any split interval technique [2] or direct sum method [13], obtaining the eigenrelation for the non-standard SL boundary value problem would require a careful examination of the asymptotic properties of the eigenfunction solutions. The function of interest here would be the function $\theta\left(\xi, \gamma_{2}\right)$, as we shall shortly see. We shall use the asymptotic approximation for $\arg \left(2 i \gamma_{2} \xi k\right)<2 \pi$, namely

$$
\begin{aligned}
\frac{M_{k, \mu}\left(2 i \gamma_{2}^{\frac{1}{2}} \xi\right)}{\Gamma(1+2 \mu)} \sim\left(2 i \gamma_{2}^{\frac{1}{2}} \xi\right)^{\frac{1}{4}} \pi^{-\frac{1}{2}} k^{-\frac{1}{4}-\mu} \cos \left\{2 \sqrt{2 i \gamma_{2}^{\frac{1}{2}} \xi k}-\pi\left(\mu+\frac{1}{4}\right)\right\} \\
\cdot\left\{1+O\left(|k|^{-\frac{1}{2}}\right)\right\}, \\
\frac{M_{-k, \mu}\left(2 i \gamma_{2}^{\frac{1}{2}} \xi\right)}{\Gamma(1+2 \mu)} \sim\left(2 i \gamma_{2}^{\frac{1}{2}} \xi\right)^{\frac{1}{4}} \pi^{-\frac{1}{2}} k^{-\frac{1}{4}-\mu} e^{\mp \pi i\left(\mu+\frac{1}{4}\right)} \\
\cdot \cos \left\{2 \sqrt{2 i \gamma_{2}^{\frac{1}{2}} \xi k} e^{ \pm \frac{1}{2} \pi i}-\pi\left(\mu+\frac{1}{4}\right)\right\} \\
\cdot\left\{1+O\left(|k|^{-\frac{1}{2}}\right)\right\} .
\end{aligned}
$$

We may then write

$$
\theta\left(\xi, \gamma_{2}\right)=\nu_{0}\left[\begin{array}{c}
\alpha_{0} \cos \left\{2 \sqrt{2 i \gamma_{2}^{\frac{1}{2}} \xi k}-\pi\left(\mu+\frac{1}{4}\right)\right\}\left\{1+O\left(|k|^{-\frac{1}{2}}\right)\right\} \\
+\alpha_{1}^{*} \cos \left\{2 \sqrt{2 i \gamma_{2}^{\frac{1}{2}} \xi k e^{ \pm \frac{1}{2} \pi i}}-\pi\left(\mu+\frac{1}{4}\right)\right\}\left\{1+O\left(|k|^{-\frac{1}{2}}\right)\right\}
\end{array}\right]
$$

where $\alpha_{1}^{*}=\alpha_{1} e^{\mp \pi i\left(\mu+\frac{1}{4}\right)}$ and $\nu_{0}=\left(2 i \gamma_{2}^{\frac{1}{2}} \xi\right) \pi^{-\frac{1}{2}} k^{-\frac{1}{4}-\mu}$.

Let us now apply the boundary conditions at $a$ and $b$ as follows:

$$
\begin{aligned}
& \theta\left(a, \gamma_{2}\right)=\nu_{0}\left[\begin{array}{c}
\alpha_{0} \cos \left\{2 \sqrt{2 i \gamma_{2}^{\frac{1}{2}} a k}-\pi\left(\mu+\frac{1}{4}\right)\right\}\left\{1+O\left(|k|^{-\frac{1}{2}}\right)\right\} \\
+\alpha_{1}^{*} \cos \left\{2 \sqrt{2 i \gamma_{2}^{\frac{1}{2}} a k e^{ \pm \frac{1}{2} \pi i}}-\pi\left(\mu+\frac{1}{4}\right)\right\}\left\{1+O\left(|k|^{-\frac{1}{2}}\right)\right\}
\end{array}\right] \\
& +n^{+}\left(\gamma_{2}\right) \phi\left(a, \gamma_{2}\right) \\
& =0 \text {, } \\
& \theta\left(b, \gamma_{2}\right)=\nu_{0}\left[\begin{array}{c}
\alpha_{0} \cos \left\{2 \sqrt{2 i \gamma_{2}^{\frac{1}{2}} b k}-\pi\left(\mu+\frac{1}{4}\right)\right\}\left\{1+O\left(|k|^{-\frac{1}{2}}\right)\right\} \\
+\alpha_{1}^{*} \cos \left\{2 \sqrt{2 i \gamma_{2}^{\frac{1}{2}} b k} e^{ \pm \frac{1}{2} \pi i}-\pi\left(\mu+\frac{1}{4}\right)\right\}\left\{1+O\left(|k|^{-\frac{1}{2}}\right)\right\}
\end{array}\right] \\
& +m^{+}\left(\gamma_{2}\right) \phi\left(b, \gamma_{2}\right) \\
& =0 \text {. }
\end{aligned}
$$


Through a careful limiting process (using (2.16) and (2.19)) we have that

$$
n^{+}\left(\gamma_{2}\right) \phi\left(a, \gamma_{2}\right) \longrightarrow 0 \text { as } \xi \longrightarrow a
$$

and

$$
m^{+}\left(\gamma_{2}\right) \phi\left(b, \gamma_{2}\right) \longrightarrow 0 \text { as } \xi \longrightarrow b .
$$

Thus for

$$
\sin \left\{2 \sqrt{2 i \gamma_{2}^{\frac{1}{2}} b k}-\pi\left(\mu+\frac{1}{4}\right)\right\}=\cos \left\{2 \sqrt{2 i \gamma_{2}^{\frac{1}{2}} b k} e^{ \pm \frac{1}{2} \pi i}-\pi\left(\mu+\frac{1}{4}\right)\right\}
$$

and

$$
\sin \left\{2 \sqrt{2 i \gamma_{2}^{\frac{1}{2}} a k}-\pi\left(\mu+\frac{1}{4}\right)\right\}=\cos \left\{2 \sqrt{2 i \gamma_{2}^{\frac{1}{2}} a k} e^{ \pm \frac{1}{2} \pi i}-\pi\left(\mu+\frac{1}{4}\right)\right\},
$$

we have from (3.4) and (3.5), that

$$
\sin \left\{2 \sqrt{2 i \gamma_{2}^{\frac{1}{2}} b k}-2 \sqrt{2 i \gamma_{2}^{\frac{1}{2}} a k}\right\}=0 .
$$

From whence we obtain,

$$
\gamma_{2}^{(p)}=\frac{p^{4} \pi^{4}}{16\{2 i k(b+a)+4 k \sqrt{a b}\}^{2}},
$$

where $p$ is the mode number.

This completes the proof of the following theorem.

Theorem. Let the SL eigenproblem (2.1) with (2.2) for the low order modes, for which the turning points are separated and none coincides with any of the end points of the interval $I_{\xi}=[a, b]$, have solutions (2.15). If

$$
\sin \left\{2 \sqrt{2 i \gamma_{2}^{\frac{1}{2}} b k}-\pi\left(\mu+\frac{1}{4}\right)\right\}=\cos \left\{2 \sqrt{2 i \gamma_{2}^{\frac{1}{2}} b k} e^{ \pm \frac{1}{2} \pi i}-\pi\left(\mu+\frac{1}{4}\right)\right\}
$$

and

$$
\sin \left\{2 \sqrt{2 i \gamma_{2}^{\frac{1}{2}} a k}-\pi\left(\mu+\frac{1}{4}\right)\right\}=\cos \left\{2 \sqrt{2 i \gamma_{2}^{\frac{1}{2}} a k} e^{ \pm \frac{1}{2} \pi i}-\pi\left(\mu+\frac{1}{4}\right)\right\},
$$

for which $\arg \left(2 i \gamma_{2} \xi k\right)<2 \pi$, then the SL eigenproblem for low order modes has the eigenrelation

$$
\gamma_{2}^{(p)}=\frac{p^{4} \pi^{4}}{16\{2 i k(b+a)+4 k \sqrt{a b}\}^{2}} .
$$

4. Concluding remarks. The matching technique in [1] resulted in eigenfunction solutions that were valid for the entire unsplit interval, and so the eigenrelation for low order modes did not require any special condition or constraints in its derivation. However, when one considers split interval techniques, which include the Titchmarsh-Weyl $m$-function technique and the direct sum method ([1] and [10), it becomes imperative that some minimal conditions for the derivation of the eigenrelations be stated. As in the theorem above, with the indicated conditions, we obtain for the first time the eigenrelation for low order modes for an SL eigenproblem with interior singularities. Our formula 
is applicable with the choice of branch cut made in the theory of atmospheric and oceanic waves. With the obvious popularity of these methods of solution partly because of their simplicity, the above approach when implemented for the $n t h$ (for $n>2$ ) order interior singularities would yield similar results. The ease of obtaining such results would also depend on an appropriate analysis on the behavior of the eigenfunction solutions, most of which are not well behaved, as is evident above.

\section{REFERENCES}

[1] Acho T.M, A matching technique and structure theorems for the Sturm-Liouville boundary value problems with interior singularities, Quaestiones Mathematicae 25(2002), 275-287 MR1931279 (2003j:34096)

[2] Acho T.M and D.P. Clemence, Sturm-Liouville eigenproblems with an interior double pole, Zeitschrift fur Angewandte Mathematik und Physik,Vol.46(1995), 459 -474. MR1335913 (96b:34037)

[3] Acho T.M and D.P. Clemence,The parameter dependent Sturm-Liouville Eigenproblem with an interior simple or double pole. Journal of the Australian Mathematical Society, ANZIAM J. 43 (2002) 1 - 13 MR1903912 (2003b:34065)

[4] Boyd J.P., Complex Co-ordinate Methods for Hydrodynamic Instabilities and Sturm-Liouville Eigenproblems with an Interior Singularity, Journal of Computational Physics, Vol. 54, No. 3, (1985) 454-471 MR782992 (86g:65148)

[5] Boyd J.P., Sturm-Liouville Eigenproblems with an Interior Pole, J. Math. Physics, 22, (8), (1981), 1575-1590. MR628532 (82k:34067)

[6] Boyd J.P., The influence of meridional shear on planetary waves. Part I: Non singular wind profiles, J. Atm. Sci. 39 (4) 756-769 (1982). MR668786 (84f:86011a)

[7] Boyd J.P., The influence of meridional shear on planetary waves. Part II: Critical latitudes, Ibid 770-790. MR668787 (84f:86011b)

[8] Bleistein N. and R.A. Handelsman, Asymptotic Expansions of Integrals, Dover Publications Inc., New York, (1986) MR863284 (89d:41049)

[9] Carrier G.F., M. Krook and C.E. Pearson, Functions of a Complex variable, McGraw Hill, New York, (1966) MR0222256 (36:5308)

[10] Coddington, E.A and Levinson, N., Theory of Ordinary Differential Equations, R.E. Krieger Publ. Co. Malabar, Fl. 1984. MR0069338 (16:1022b)

[11] Dickinson, R.E., Planetary Rossby wave propagating vertically through weak westerly wind wave guides, J. Atm. Sci. 25, (1968), 984-1002

[12] Everitt, W.N. and Zettl, A., Sturm-Liouville differential operators in direct sum spaces, Rocky Mountain J. Math. (1986) 497-516. MR862277 (88a:34025)

[13] Everitt W.N.; J. Gunson and A. Zettl, Some Comments on Sturm-Liouville Eigenvalue problems with Interior Singularities, Zeitschrift fur Angewandte Mathematik und Physik, Vol 38 (1987), 813-838. MR 928586 (89h:34022)

[14] Friedman B.; Principles of Applied Mathematics, John Wiley and Sons Inc., (1966).

[15] Nayfeh, A.H.; Perturbation Methods, Willey, New York (1973). MR0404788 (53:8588)

[16] Olver F.W.J. Asymptotic and Special Functions ; Academic Press, New York 1973. MR0435697 (55:8655)

[17] Shibov M.A, High-energy asymptotics of resonances for three dimensional Schrodinger operator with screened Coulomb potential, J.Math.Phys.35, no 2, (1994),656 -679. MR.1257539 (94m:81128)

[18] Simmons, A.J., Planetary-scale disturbances in the polar winter stratosphere, Quart. J. R. Met. Soc. 100, (1974), 76-108.

[19] Slater L.J. Confluent Hypergeometric Functions, Cambridge University Press 1960. MR0107026 $(21: 5753)$ 